\title{
A preliminary study of patient comfort associated with customised mouthguards
}

\author{
C McClelland, M Kinirons, L Geary
}

\begin{abstract}
Objective-To compare patient perception of custom made mouthguards of ideal and less than ideal designs in terms of their comfort and "wearability".
\end{abstract}

Method-A mouthguard of ideal design (A) and one incorporating common design faults of underextension and unadjusted occlusion (B) were provided for 22 active sportsmen and women. They were not informed of the details of the design or the status of the protector. Half the participants were asked to wear mouthguard $A$ first and the other half wore B first, each worn for one hour on two consecutive nights. Questionnaires were used to evaluate and rate the comfort and wearability of each mouthguard.

Results-Eighteen people completed the study. The ideal appliance was rated as significantly more retentive and comfortable overall and specifically was more comfortable to lips, gums, and tongue. It was also recognised as being less bulky, less likely to keep the teeth apart, or to cause pain in the jaw muscles.

Conclusions-Comfort is likely to be increased if mouthguards are extended labially to within $2 \mathrm{~mm}$ of the vestibular reflection, adjusted to allow even occlusal contact, rounded at the buccal peripheries, and tapered at the palatal edges.

(Br F Sports Med 1999;33:186-189)

Keywords: mouthguards; design faults; teeth; jaws; safety

Injury to the upper central incisors and soft tissue lacerations or bruising are the most commonly sustained orofacial injuries during sports. ${ }^{1-3}$ There has been no system of overall monitoring of the incidence of dental injury in sports, although some studies have surveyed the occurrence of orofacial injury in particular sports. The risks in boxing, football, and rugby are well recognised. ${ }^{45}$ It has been found that $63 \%$ of rugby players ${ }^{6}$ and $62 \%$ of international hockey players ${ }^{7}$ have sustained dental injuries during sport. At least one in ten ice hockey players have lost a tooth, ${ }^{8}$ and, in one study of basketball players, $31 \%$ were found to have sustained an oral injury during a single season. ${ }^{9}$ The use of mouthguards in sport has resulted in a decrease in the number of injuries and an increased interest in the issue. ${ }^{210}$ It has been estimated that $50 \%$ of dental injuries in adolescents in Britain are sustained during organised sport at school. ${ }^{11}$ This represents a significant number of traumatic injuries, which can cause considerable pain and have functional and aesthetic implications, which are preventable or at least could be limited by the use of mouthguards. If we consider that by the age of $15,27 \%$ of children in Northern Ireland have sustained damage to their permanent incisors, a significant number of these will be sustained through sporting activities. ${ }^{12}$

The mouthguard acts by absorbing some of the energy imparted at the site of impact and by dissipating the remaining energy. If the force is very high, its detrimental effect can be reduced. ${ }^{1}$ The mouthguard holds the soft tissues of the lips and cheeks away from the sharp edges of the teeth and thereby decreases the risk of laceration and bruising. ${ }^{813}{ }^{14}$ Forces from direct frontal blows to the mouth, which could fracture or cause dislocation of the anterior maxillary teeth, are cushioned and redistributed, and violent contacts of the teeth in opposing arches are also prevented. ${ }^{13}$ The mouthguard also protects against jaw fractures by absorbing some of the traumatic energy and provides braced support for the mandible to protect against fracture of the angle of the mandible or unsupported angle of the condyle. ${ }^{13}$ The bite planes prevent full post translation of the condyles subsequent to an impact to the mandible from either in front or below. The condyle is thus prevented from being displaced upwards and backwards against the base of the skull.

Most athletes appreciate that wearing a mouthguard provides protection to the jaws and teeth, but many choose not to wear any such devices. Patient compliance is critical if further reductions in the incidence of these sports injuries is to occur. Compliance is highly influenced by the comfort of the device and may be related to its fit and extension. ${ }^{11}$ Patient perception of the mouthguard is important as this largely determines their compliance and enthusiasm. ${ }^{15}$ The mouthguard should be comfortable, retentive, allow normal breathing and speech, and not impinge on the soft tissues $^{11}$. It should not cause nausea or a dry mouth. ${ }^{16}$ There are no regulatory bodies to enforce these requirements, and, despite these guidelines, there are mouthguards in use that do not conform to the recommended design features, in terms of extension, thickness, shaping of the peripheries, and occlusal adjustment. Can changes in these factors affect patient acceptability of a mouthguard? This preliminary study aims to compare patient perception of custom made mouthguards of ideal and less than ideal designs in terms of their comfort and "wearability".
Accepted for publication 3 February 1999 
Methods

Twenty two subjects who were active in sports and between the ages of 20 and 26 were recruited to the study. Details of their participation in sport and their experience of wearing mouthguards were recorded. Upper and lower arch alginate impressions were taken of each patient taking care to record the full depth of the upper labial sulcus. A wax interocclusal record was taken. The impressions were cast in $50 \%$ plaster of Paris and 50\% Hydrocal. A fully extended occlusally adjusted mouthguard conforming to the ideal design requirements ${ }^{16}$ was fabricated for each patient as follows. The outline of the mouthguard was marked on the upper models $2 \mathrm{~mm}$ from the full depth of the labial sulcus and $1 \mathrm{~mm}$ from the palatal surface of the second molars, terminating at the distal of the second molars and covering one third of the palate. A post dam was engraved on to the models and the models were coated in sodium alginate. A $3 \mathrm{~mm}$ blank of polyvinylacetatepolyethylene was adapted to the model using a Drufomat-U compression forming device. A second $3 \mathrm{~mm}$ sheet of acrylic was pressure formed on top of the first layer and the peripheries trimmed and rounded labially and bucally and tapered palatally. The models were mounted on an articulator, and the mouthguard was adjusted by grinding the occlusal acrylic until even contact was achieved.

In addition, a mouthguard incorporating common design faults was provided for each person. It was similar to the ideal design but the labial extension as measured from the the gingival margin of the upper left central incisor was half that of the former and no occlusal adjustment was carried out nor were the peripheries rounded and finished. Figure 1 shows the labial outlines of the two types of appliances. The mouthguards were labelled A (ideal) and B (non-ideal) but the subjects were not informed of the details of the design or the status of the protector. Half were asked to wear mouthguard A first and the other half wore B first. While inactive, they wore each of them, for two consecutive nights, for one hour each night (the average length of a training session or match). Questionnaires were administered which allowed the subjects to evaluate and rate the comfort and wearability of each mouthguard, as outlined in table 1 . They were asked to rate the comfort, ease of breathing, and retention of the mouthguards on a scale of 1 to 10,1 being the poorest score and 10 the highest, and the poles of the scales were labelled accordingly. They were also asked to rate individually the comfort scores of particular areas. The questionnaire also included general questions about the wearability, tightness, bulk, and whether it held the teeth too far apart or caused any pain in the jaws or felt uneven to bite on. The participants were asked to assess the ease of speech and swallowing when wearing the mouthguard and whether it caused a feeling of mouth dryness or nausea. The ratings were transferred to coding sheets and these data were entered on to the NCSS program for Windows run on an IBM personal computer to facilitate analysis. The frequencies of responses

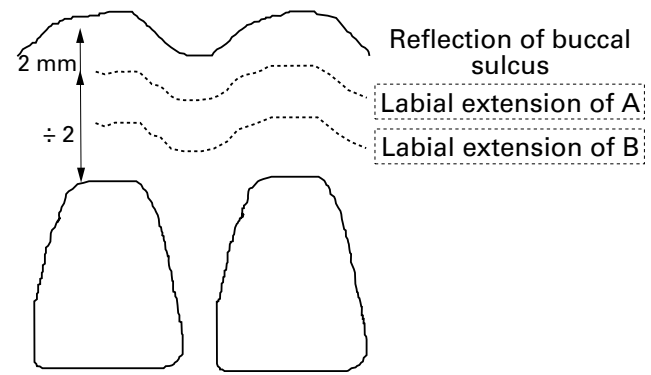

Figure 1 Labial extension of mouthguard $A$ and $B$.

Table 1 Comfort, retention, and ease of breathing for appliance $A$ (ideal) compared with appliance $B$ (non-ideal)

\begin{tabular}{lllll}
\hline Protector & Median & Range & $Z^{\star}$ & $p$ \\
\hline Overall comfort & & & & \\
A & 8 & $4-10$ & & \\
B & 6 & $1-9$ & 2.86 & 0.004 \\
Lip comfort & & & & \\
A & 9 & $6-10$ & & \\
B & 8 & $2-10$ & 2.39 & 0.017 \\
Gum comfort & & & & \\
A & 9 & $5-10$ & & \\
B & 7 & $3-9$ & 2.80 & 0.005 \\
Tongue comfort & & & & \\
A & 8 & $4-10$ & & \\
B & 7 & $1-10$ & 2.42 & 0.015 \\
Tooth comfort & 8 & $4-10$ & & \\
A & 7 & $2-10$ & 0.92 & 0.355 \\
B & & & & \\
Retention & 10 & $7-10$ & & \\
A & 8 & $2-10$ & 2.36 & 0.018 \\
B & & & & \\
Ease of breathing & & $6-10$ & & \\
A & 9 & $4-10$ & 2.48 & 0.013 \\
B & 8 & & &
\end{tabular}

*Wilcoxon's signed rank test.

were calculated and differences between the two appliances were tested for statistical significance using Wilcoxon's signed rank test and McNemars $\chi^{2}$ test as appropriate.

\section{Results}

Questionnaires were returned by 18 out of 22 subjects (nine men and nine women). Seven respondents played hockey, seven played squash, six played football, four played rugby, and two were boxers. They played an average of 2.5 times per week. Before the study, 15 of the participants had experience of wearing a mouthguard, three a stock mouthguard, eight a self adapted mouthguard, and four a customised mouthguard made by a dental practitioner. Nine of the 15 continued to use their mouthguard. Table 1 gives the results on comfort, retention, and ease of breathing. Overall comfort was significantly better for appliance A and it was considered to be more retentive. Appliance B was described as significantly less comfortable to the lips, gums, and tongue, while the comfort around the teeth was evenly rated between appliances.

Table 2 gives the responses on wearability of the appliances. Only one participant complained that the mouthguard felt too loose, referring to type B. Significantly more participants described $\mathrm{B}$ as feeling bulky. A significant number of participants felt that $B$ held the mouth open and the teeth apart whereas A did not. A significantly larger number of subjects complained that B caused pain in the muscles of the jaw compared 
Table 2 Wearability of appliance $A$ (ideal design) compared with appliance $B$ (non-ideal)

\begin{tabular}{lrrll}
\hline Protector & No & Yes & $\chi^{2}$ & $p^{*}$ \\
\hline $\begin{array}{c}\text { Too Loose } \\
\text { A }\end{array}$ & 18 & 0 & NA & NA \\
B & 19 & 1 & & \\
$\begin{array}{c}\text { Too tight } \\
\text { A }\end{array}$ & 13 & 5 & 1.00 & 0.317 \\
B & 12 & 6 & & \\
Bulky A & 16 & 2 & 10.29 & 0.001 \\
B & 4 & 14 & & \\
Small/thin & & & & \\
A & 17 & 1 & 0.33 & 0.56 \\
B & 16 & 2 & & \\
Held mouth open & & & & \\
A & 17 & 1 & 7.36 & 0.007 \\
B & 8 & 10 & & \\
Held teeth apart & & & & \\
A & 17 & 1 & 5.44 & 0.019 \\
B & 11 & 7 & & \\
Pain in jaw muscles & & & & \\
A & 17 & 1 & 8.00 & 0.005 \\
B & 9 & 9 & & \\
Uneven bite & & & & \\
A & 13 & 5 & 0.82 & 0.366 \\
B & 10 & 8 & & \\
M & & & &
\end{tabular}

${ }^{\star}$ McNemars test.

The numbers of respondents in each category are shown. NA not applicable.

Table 3 Comparison of effect of mouthguards $A$ (ideal design) and $B$ (non-ideal) on ease of speech and swallowing, mouth dryness, and nausea

\begin{tabular}{lrrrrl}
\hline Protector & No & A little & A lot & $Z^{\star}$ & \multicolumn{1}{l}{$p$} \\
\hline Interferes with speech & & & & & \\
A & 1 & 14 & 3 & 2.69 & 0.007 \\
B & 0 & 7 & 11 & & \\
Interferes with swallowing & & & & \\
A & 11 & 7 & 0 & 2.13 & 0.033 \\
B & 8 & 6 & 4 & & \\
Causes a dry mouth & & & & & \\
A & 11 & 7 & 0 & 1.00 & 0.317 \\
B & 13 & 5 & 0 & & \\
Causes nausea & & & & & \\
A & 16 & 2 & 0 & 2.32 & 0.020 \\
B & 9 & 8 & 1 & & \\
\hline
\end{tabular}

*Wilcoxon's signed rank test.

The numbers of respondents in each category are shown.

with mouthguard A. Only seven of the 18 participants were able to rate correctly both the the occlusal adjustment made to mouthguard $\mathrm{A}$ and the lack of adjustment to B.

Table 3 presents the results on ease of speech and swallowing when wearing the mouthguard and whether it caused feelings of mouth dryness or nausea. Most participants were critical that the mouthguards interfered with speech to some degree but this was significantly more apparent for appliance B. Most responded that the mouthguards interfered with swallowing a little or not at all and there was no significant difference between groups. Nausea was reported significantly more frequently for mouthguard B. Of the nine participants complaining of nausea when wearing appliance $\mathrm{B}$, eight indicated that the mouthguard was bulky. Similarly, of the 11 who reported major speech difficulty, 10 complained of bulk.

\section{Discussion}

Many custom made mouthguards fall short of the ideal specifications because of their extension, thickness, palatal coverage, occlusal adjustment, and shaping of the margins. No previous study has examined how patient comfort is influenced by variation in the design and fabrication of a custom made mouthguard. Mouthguard A was more acceptable and preferable to mouthguard B in terms of comfort. It was described as bulky by significantly fewer respondents and prompted fewer complaints that it propped the mouth open, held the teeth apart, or caused pain in the jaw muscles while being worn. It caused less interference with speech and nausea.

How does occlusal adjustment, peripheral shaping, and labial extension account for these variations in comfort? Tapering the palatal peripheries and rounding the labial and buccal peripheries reduces the prominent abrupt edges. Comparison of the comfort ratings awarded to the different designs of mouthguard relating to the lips, gums, and tongue indicates that this peripheral trimming is discernible to patients wearing the mouthguards. Rounding of the labial edges of mouthguard A improved the comfort of the device to the lips and gums. Labial comfort is also likely to be influenced by the labial extension of the mouthguard. Optimum extension-that is, continuing the flange to within $2 \mathrm{~mm}$ of the vestibular reflection-makes the edge of the mouthguard less prominent to the lip. Tapering of the palatal peripheries decreases the lingual bulk and allows the edge of the mouthguard to be less obtrusive to the tongue. As would be expected, the mouthguards were equally comfortable to the teeth, as the procedure for adaptation of the acrylic to the models did not vary. Peripheral shaping has no effect on the protective efficacy of the device, therefore it is performed purely to improve patient acceptability. The results indicate that the time and effort invested in this procedure are justified.

A symptom of lack of occlusal adjustment is a feeling of the mouth being propped open and was evident with mouthguard B. Most authors agree that occlusal adjustment is indicated to provide even occlusal contact and thus even distribution of forces and widespread dissipation of energy when the jaws are forced together. ${ }^{17}$ This study also indicates that occlusal adjustment has an important influence over comfort of the mouthguard and patient acceptance. Lack of peripheral shaping and occlusal adjustment results in the mouthguard feeling bulky in the mouth, a complaint made much more frequently about mouthguard B. A sensation of bulkiness may be associated with nausea and this is reflected in the results of this investigation. Complaints of bulkiness were associated with a higher incidence of nausea when wearing the mouthguard. Mouthguard B prompted significantly greater complaints with regard to interference with speech and this was related to a general feeling of bulkiness, which may be due to lack of peripheral and occlusal adjustment ${ }^{1819}$ or to the prominence of the mouthguard to the tongue because of lack of palatal trimming and tapering. In the present study, all three design features were incorporated in each ideal appliance and omitted in each non-ideal appliance. We do not know the effect of each separate feature and this will be asessed in the future. 
On the basis of this initial study it appears that mouthguards should be: (a) extended labially to within $2 \mathrm{~mm}$ of the vestibular reflection; (b) adjusted to allow even occlusal contact (in the laboratory and at the chairside until the patient reports that the guard is even to bite on); (c) rounded at the buccal peripheries and tapered at the palatal edges.

MK initiated and drew up the protocol for the study. LG further developed the protocol and provided technical expertise in the construction of the appliances. CM conducted the literature review and the final study design, and also drew up, distributed, and collected the questionnaires. CM and MK conducted the data analysis and produced the preliminary drafts of the paper. MK co-ordinated and produced the final version of the paper. Guarantor: MK.

1 Chapman PJ. The bimaxillary mouthguard: a preliminary report of use in contact sports. Aust Dent F 1986;31:200-8.

2 Jennings DC. Injuries sustained by users and non-users of gum shields in local rugby union. Br F Sports Med 1990;24: gum shield.

3 Stockwell AJ. Incidence of dental trauma in the Western Australian School Dental Service. Community Dent Oral Epiemiol 1988;16:294-8.

4 Turner CH. Mouthords. Br Dent f 1977;143:82-6.

4 Turner CH. Mouthguards. Br Dent $\mathcal{F}_{1977,143: 82-6 .}$. and the use of mouthguards in contact team sports. Br Dent f 1994;176:310-14
6 Hawke JE, Nicholls NK. Dental injuries in rugby football. $N$ $Z$ Dent 7 1969;65:173-5.

7 Bolhuis JHA, Leurs JMM, Flogel GE. Dental and facial injuries in international field hockey. Br F Sports Med 1987; 21:174-7.

8 Wei SH. Prevention of injury to the anterior teeth. Int Dent $\mathcal{F}$ 1974;24:30-44.

9 Nowjack-Raymer RE, Gift HC. Use of mouthguards and headgear in organised sports by school-aged children. Public Health Reports 1996;111:82-6.

10 Kay EJ, Kakarla P, Macleod DAD, et al. Oro-facial and dental injuries in club union players. Br f Sports Med 1990;24: 271-3.

11 Stenger JM, Lawson EA, Wright JM, et al. Mouthguards: protection against shock to head, neck and teeth. $\mathcal{F}$ Am Dent Assoc 1964;69:273-81.

12 O'Brien M. Children's dental health in the UK 1993. London: HMSO, 1994:79-81.

13 Andreasen JO. Traumatic injuries to the teeth. Copenhagen: Munksgaard, 1981.

14 Johnsen DC, Winters JE. Prevention of intraoral trauma in sports. Dent Clin North Am 1991;35:657-66.

15 Seals RR, Morrow RM, Kuebker WA, et al. An evaluation of mouthguard programs in Texas high school football. $\mathcal{f} \mathrm{Am}$ Dent Assoc 1985;110:904-9.

16 Chapman PJ. Mouthguards and the role of the sporting team dentists. Aust Dent F 1989;34:36-43.

17 Upton N. Mouthguards, an evaluation of two types for rugby players. Br F Sports Med 1985;19:89-92.

18 Bureau of health education. Mouthguards and sports team dentists. F Am Dent Assoc 1984;109:84-7.

19 Chapman PJ. Orofacial injuries and mouthguards: A study of the 1985 wallabies. Br f Sports Med 1985;19:93-5. 1991; 10:463-8.

Take home message

Comfort and general wearability of mouthguards will be improved if they are customised, adequately extended, and occlusally accommodated. Patient compliance in wearing the mouthguard may improve as a result.

\begin{tabular}{|c|c|c|}
\hline \multicolumn{3}{|c|}{ BASM Merchandise 1999} \\
\hline Ties & $\begin{array}{l}\text { Single motif } \\
\text { Multi motif }\end{array}$ & $\begin{array}{l}£ 6+£ 1.50 \mathrm{p} \& \mathrm{p} \\
£ 6+£ 1.50 \mathrm{p} \& \mathrm{p}\end{array}$ \\
\hline Blazer badge & $\begin{array}{l}\text { Wire - } 4 \text { inches high } \\
\text { Wire - } 3 \text { inches high }\end{array}$ & $\begin{array}{l}£ 5+£ 1.50 \mathrm{p} \& \mathrm{p} \\
£ 5+£ 1.50 \mathrm{p} \& \mathrm{p}\end{array}$ \\
\hline \multicolumn{3}{|c|}{ New stock to order } \\
\hline Sweaters & $\begin{array}{l}\text { Lambswool fine knit, V-neck or round } \\
\text { neck with small motif. Machine washable. } \\
\text { State colour and chest size required. }\end{array}$ & $£ 32+£ 3$ p\&p \\
\hline Sweatshirts & $\begin{array}{l}\text { With small motif. } \\
\text { State colour and chest size required. }\end{array}$ & $£ 25+£^{3} \mathrm{p} \& \mathrm{p}$ \\
\hline Polo shirts & \multicolumn{2}{|c|}{$\begin{array}{l}\text { State colour and chest size required. } \\
\text { Some education polo shirts in cream } \\
\text { and grey are also available at the same price. }\end{array}$} \\
\hline $\begin{array}{l}\text { Send orders t } \\
\text { Birch Lea, } 67 \\
\text { UK. (Tel: } 017\end{array}$ & $\begin{array}{l}\text { hn H Clegg JP BSc LDS RCS Eng, Hon Se } \\
\text { ingfield Lane, Eccleston, St Helens, Mersey } \\
\text { 28198) }\end{array}$ & $\begin{array}{l}\text { cretary, } \\
\text { yside WA10 5HB, }\end{array}$ \\
\hline
\end{tabular}

